\title{
Books Received at the Editorial Office
}

Appearance of a title in this list does not preclude the possibility that a review may appear in a later issue.

Gregory Bock, Geralyn M. Collins: Molecular Approaches to Human Polygenic Disease. Ciba Fdn Symp. 130. Wiley, Chichester 1987. IX+ 274 pp.; E28.95. ISBN 0-471-91096-1.

Fred A. Crawford: Current Heart Valve Prostheses. Cardiac Surgery. State of the Art. Reviews. Han-ley \& Belfus, Philadelphia 1987. VIII + 143-326 pp.; E22.95. ISSN 0887-9850.

Barry H. Greenberg, Edward Murphy: Valvular Heart Disease. PSG, Littleton 1987. IX+ 296 pp.; E38.50. ISBN 0-88416-472-1.

Grady L. Hallman, Denton A. Cooley: Surgical Treatment of Congenital Heart Disease. Lea \& Febiger, Philadelphia 1987. 235 pp.; US\$ 45.00. ISBN 0-8121-1069-2.

Stephen Hunyor: Cardiovascular Drug Therapy. Williams \& Wilkins, Baltimore 1987. 309 pp.; US\$ 60.00. ISBN 0-86433-005-7.

John B. Kostis, Eugene A. DeFelice: Angiotensin Converting Enzyme Inhibitors. Liss, New York 1987.

XIII + 285 pp.; US\$ 39.50. ISBN 0-8451-4240-2.

Wade C. Lamberth, Donald B. Doty: Peripheral Vascular Surgery. Year Book Medical Publishers, Chicago 1987. XVI + 341 pp.; E55.50. ISBN 0-8151-5324-4.

Henry J.L. Marriott: Rhythm Quizlets. Self Assessment. Lea \& Febiger, Philadelphia 1987. X+ 189 pp. ISBN 0-8121-1110-9.

J.L. Mehta: Thrombosis and Platelets in Myocardial Ischemia. Cardiovascular Clinics. Davis, Philadelphia 1987. XV + 294 pp. ISBN 0-8036-6051-0.

L.W. Pickle, Th.J. Mason: Atlas of US Cancer Mortality Among Whites: 1950-1980. US Department of Health and Human Services, Bethesda 1987.

IV + 184pp.;US\$ 24.00. ISBN 017-042-00196-0. 